\title{
Maximum Concentration Normalized by Surface Area
}

National Cancer Institute

\section{Source}

National Cancer Institute. Maximum Concentration Normalized by Surface Area. NCI

Thesaurus. Code C92372.

The maximum concentration occurring at T max, divided by the surface area. 